(1989); Y. Uehara, Y. U. Murakami, Y. Sugimoto, S. Mizuno, Cancer Res. 49, 780 (1989).

24. Coculture and analysis were performed as de scribed for Fig. 4A.

25. The relative tyrosine phosphate content of $p p 30$ for each of the lysates in Fig. 5A was determined by laser densitometry to be $31 \%$ (lane 1); $100 \%$ (lane 2); 326\% (lane 3); 77\% (lane 4); 165\% (lane 5 ); and $133 \%$ (lane 6 ).

26. In Fig. 5D, total cell number and viability (\%) determined at harvest for each of the culture conditions, were $10^{7}(97 \%)$ in media alone; $5.5 \times$ $10^{6}(81 \%)$ with HIV; and $7.5 \times 10^{6}(91 \%)$ with HIV + herbimycin $\mathrm{A}$.

27. J. E. K. Hildreth and R. J. Orentas, Science 244
1075 (1989); G. Pantaleo et al., J. Exp. Med. 173, 511 (1991).

28. D. Zagury et al., Science 231, 850 (1986); J. S. McDougal et al., J. Immunol. 135, 3151 (1985); S D. Gowda, B. S. Stein, N. Mohagheghpour, C. J. Benike, E. G. Engleman, ibid. 142, 773 (1988); P. N. Fultz, J. Virol. 65, 4902 (1991)

29. L. Ratner et al., Nature 313,277 (1985).

30. We thank A. S. Fauci and R. Klausner for the critical review of this manuscript, $C$. June for anti-CD3 monoclonal antibodies, Genentech for soluble CD4, and $\mathrm{M}$. Rust for valuable assistance in the preparation of this manuscript.

2 December 1991; accepted 20 February 1992

\title{
Observational Learning in Octopus vulgaris
}

\section{Graziano Fiorito and Pietro Scotto}

Untrained Octopus vulgaris (observers) were allowed to watch conditioned Octopus (demonstrators) perform the task of selecting one of two objects that were presented simultaneously and differed only in color. After being placed in isolation, the observers, in a similar test, consistently selected the same object as did the demonstrators. This learning by observation occurred irrespective of the object chosen by the demonstrators as the positive choice and was more rapid than the learning that occurred during the conditioning of animals. The task was performed correctly without significant errors and further conditioning for 5 days. These results show that observational learning can occur in invertebrates.

Octopuses, like many other invertebrate species, are capable of a learned change in behavior as a result of experience (1-4), demonstrating that Octopus can integrate information to produce adaptive behavioral patterns (5). Among vertebrates living in social groups, learning can be facilitated by observation of another member of the same species (conspecific) performing a behavioral act $(6,7)$.

Octopus vulgaris does not have social habits, which implies that it has little experience observing the behavior of conspecifics. However, some cephalopods are social, and specialized behavioral patterns are exhibited when octopuses come in contact with each other (8). Therefore, we tested whether octopuses can learn to perform a task by observation of other trained octopuses.

Individuals of Octopus vulgaris were conditioned to discriminate between two stimuli that were identical in shape and size but differed in color (9). Training was followed by a session of trials while an untrained Octopus visually observed the choices made by the conditioned demonstrator. Experiments to investigate the ability of Octopus vulgaris (10) to learn by observation were conducted in three phases: (i) training of demonstrators, (ii) observation of the task by untrained octopuses, and (iii) testing of observers.

G. Fiorito, Laboratorio di Neurobiologia Stazione Zoologica A. Dohrn di Napoli, I-80121 Napoli, Italy.

P. Scotto, Cattedra di Fisiologia Umana, Università di Reggio Calabria, I-88100 Catanzaro, Italy.
Training of demonstrators (11) was realized by a series of trials where two balls were presented to the animal. We selected one ball to be the correct choice. When the animal attacked the correct ball it was rewarded, and each attack of the incorrect object was punished. The training of a demonstrator was complete when the animal made no mistakes in five trials.

Two groups (red balls and white balls) of demonstrators were trained. For the red group, the red ball was considered to be the correct choice. Its selection was rewarded with a small piece of fish attached to the side of the ball that was not visible to the animal. Selection of the white ball was punished by an electric shock (9). For the second group of octopuses the conditions were reversed. For the octopuses $(n=30)$ conditioned to choose the red ball, full training was reached at $16.83 \pm 1.35$ trials (mean \pm standard error of the mean). The white group $(n=14)$ was trained after $21.50 \pm 1.46$ trials. Full training was reached at a significantly different number of trials for the two groups (Student $t$ test $=$ 2.11, $\mathrm{df}=42, P<0.05)(12)$.

During the observational phase, an untrained Octopus vulgaris housed in the tank adjacent to its demonstrator observed four trials during which the demonstrator attacked the ball it had been taught to attack (Fig. 1). During this phase no errors were detected in the demonstrator groups (Fig. 2, $A$ and $C$ ), even though they were no longer rewarded for making the correct response.
From the analysis of videorecordings we noted that observer octopuses increased their attention (13) during each of the four trials. In particular, we noted that the observers followed the action patterns of their demonstrators with movements of the head and eyes. When these movements during observational trials were compared with those seen during intertrial times, we detected a significative increase in their number [Wilcoxon matched-pairs test: $z=5.23, P<0.01, n=(44$ observers minus 2 null differences) $=42$ ]. The observers also spent more time outside of their homes and displayed other behavioral patterns that have been recognized for Octopus vulgaris when in the presence of a conspecific $(8,13)$.

For the testing phase, observer octopuses were tested (14) with a session of five trials of simultaneous presentation of both white and red balls randomly positioned. No reward or punishment was given at this time for any choice made. The Octopus vulgaris $(n=30)$ that observed demonstrators attacking the red ball chose the red ball significantly more often than they did the white ball [129 red, 13 white in 150 trials; $\chi^{2}=90, P<0.01$ (Fig. 2B)]. Animals $(n=14)$ that observed demonstrators of the white group chose the white ball $\left[7\right.$ red, 49 white in 70 trials; $\chi^{2} \approx 28, P<$ 0.01 (Fig. 2D)].

During the testing phase, the red and white observers made 14 and $30 \%$ of cumulative errors, respectively. However, for both groups of observers if one considers only the number of attacks to the wrong ball, errors were $\sim 9 \%$. Failure to attack

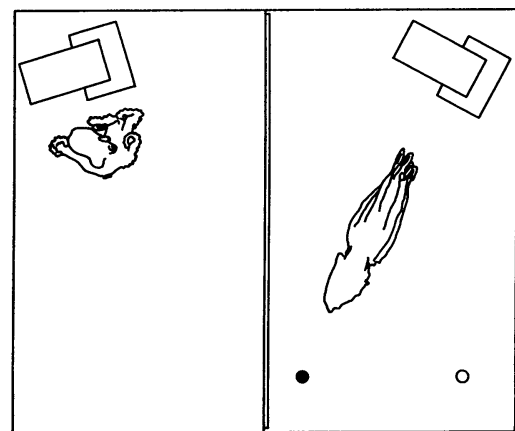

Fig. 1. Schematic of the experimental apparatus and protocol. An Octopus vulgaris is shown (right side of the figure) attacking a ball (the red one) and acting as a demonstrator for the other animal (observer, left side) that is standing outside of its home and watching its conspecific during the whole session through a transparent wall. Each tank had an independent supply of running water. Octopuses were allowed to visually interact for 2 hours before the start of the observational phase. Mean duration of the trials, which depended on the demonstrator's performances, was $40 \mathrm{~s}$, and intertrial intervals were fixed at $5 \mathrm{~min}$. 
Table 1. Learning retention by observers. Mean \pm standard deviation from five experiments with red and white groups of observers and untrained octopuses (12) at 1 and 5 days after the observational phase. The preference for red in untrained animals during free choice experiments was significant at day 5 .

\begin{tabular}{|c|c|c|c|c|c|}
\hline & \multirow{2}{*}{$n^{\star}$} & \multicolumn{2}{|c|}{ Day 1} & \multicolumn{2}{|c|}{ Day 5} \\
\hline & & Red & White & Red & White \\
\hline Observers (red) & 16 & $\begin{array}{c}4.31 \pm 1.01 \\
(4.30) \dagger\end{array}$ & $\begin{array}{c}0.31 \pm 0.60 \\
(0.43)\end{array}$ & $\begin{array}{c}3.88 \pm 1.55 \\
(4.30)\end{array}$ & $\begin{array}{c}0.50 \pm 0.63 \\
(0.43)\end{array}$ \\
\hline Observers (white) & 10 & $\begin{array}{c}0.40 \pm 0.52 \\
(0.50)\end{array}$ & $\begin{array}{c}4.10 \pm 0.58 \\
(3.50)\end{array}$ & $\begin{array}{c}0.50 \pm 0.53 \\
(0.50)\end{array}$ & $\begin{array}{c}3.70 \pm 0.82 \\
(3.50)\end{array}$ \\
\hline Untrained & 18 & $2.11 \pm 1.23$ & $1.94 \pm 0.94$ & $3.22 \pm 1.31$ & $0.89 \pm 0.96$ \\
\hline
\end{tabular}

${ }^{*} n$, number of animals tested. TThe values from the testing phase are shown in parentheses.

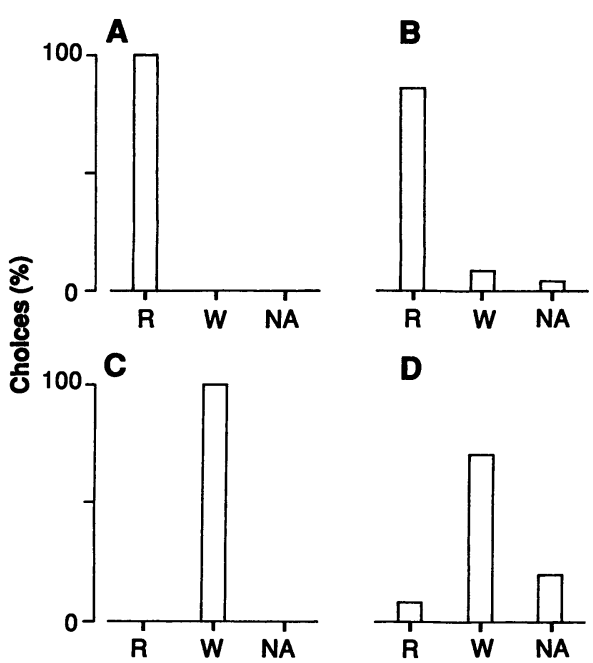

Fig. 2. Choices expressed as percent of the total choices made by demonstrators during the observational phase of the experiment and those of observers during the testing phase. (A) Red group demonstrators $(n=30)$; (B) red group observers $(n=30)$; (C) white group demonstrators $(n=14)$; and (D) white group observers $(n=14)$. R, red; W, white; NA, no attack.

(Fig. 2, B and D: NA) did not correlate with choices in preceding trials. The randomness of this event does not favor the lack of discrimination between objects or the failure of learning, but it may be related to a lack of reward after each trial.

These results suggest that untrained Octopus vulgaris can learn a task by observing, for a short period of time (four trials), the behavior of a conspecific. Observational learning was significantly faster than the learning of the demonstrators trained with classical conditioning techniques ( $\sim 16$ trials for red and $\sim 21$ for white demonstrators). After four conditioning trials success rates of $51 \%$ for red and $51 \%$ for white group were attained, whereas four trials of observation with demonstrators led to corresponding success rates of 86 and $70 \%$ (15).

Learning appeared stable when its retention by some randomly selected observers ( $n=16$ red group; $n=10$ white group) was tested on five consecutive days, with a session of five trials a day. The procedure used was the same as for the testing phase of the experiment. There was no statistically significant difference between the percentages of correct responses of the octopuses immediately after the observational phase and 5 days after its end (red, $81 \%$ versus $81 \%$; white, $70 \%$ versus $81 \%$ ) (Table 1). This indicated that the level of learning of the discrimination task that Octopus vulgaris observed is stable over time, any innate individual preference (12) being suppressed by the learned one. The significant increase in the preference for red by the untrained octopuses (from 42 to $64 \%$ ) may indicate a preference for some property of the red ball, which if true may partly account for the larger error rates in the white observer group during the testing phase of the experiment. The rapid acquisition and the stability indicate that observational learning in Octopus vulgaris is a powerful mechanism of learning, compared both to conditioning (training of demonstrators) and trial-and-error learning (4).

Copying of a model (16) is reported for humans (17) and vertebrates (18), and it has been considered preliminary to conceptual thought; in this sense it appears related to the cognitive abilities of the animal learning system (19).

The evidence presented in support of observational learning in Octopus vulgaris is particularly interesting because of the highly developed invertebrate brain of this species, with its intriguing analogies with the neural organization of vertebrate brains $(2$, 20). Questions still arise about the function of such learning in the natural environment of octopuses.

\section{REFERENCES AND NOTES}

$\rightarrow$ J. Z. Young, Philos, Trans, R. Soc London Ser. B 159, 565 (1964); H. Maldonado, J. Theor. Biol. 5 470 (1963); M. J. Wells, Symp. Soc. Exp. Biol. 20, 477 (1966); J. Z. Young, The Anatomy of the Nervous System of Octopus vulgaris (Oxford Univ. Press, London, 1971), p. 1; P. Crancher, M. G. King, A Bennett, R. B. Montgomery, J. Exp. Anal. Behav. 17, 359 (1972).
2. J. Z. Young, Biol. Rev. 36, 32 (1961).

3. W. G. Quinn, in The Biology of Learning. P. Marler and H. S. Terrace, Eds. (Springer-Verlag, Berlin, 1984), p. 197.

4. G. Fiorito, C. von Planta, P. Scotto, Behav. Neural Biol. 53, 217 (1990).

5. P. R. Boyle, in The Mollusca, vol. 9, Neurobiology and Behavior, A. O. Dennis Willows, Ed. (Academic Press, New York, 1986), p. 1

6. M. Robert, Psychol. Rec. 40, 249 (1990)

7. B. Pallaud, Behav. Processes 9, 381 (1984).

8. J. L. Yarnall, Anim. Behav. 17, 747 (1969); A Packard and G. D. Sanders, ibid. 19, 780 (1971); P. R. Boyle, ibid. 28, 1123 (1980).

9. Smooth plastic balls, white and red, were used as stimuli to be discriminated. Each ball was $3 \mathrm{~cm}$ in diameter, suspended on translucent nylon sticks, and supplied with a pair of electrodes (coming out from the body of the ball for $5 \mathrm{~mm}$ ) carrying $12 \mathrm{~V}$ of ac current when activated by a foot switch. We checked from videorecordings that balls could be easily distinguished from the background of the tank either on full color or on black and white monitors. Because Octopus vulgaris is color blind [J. B. Messenger, J. Exp. Biol. 70, 49 (1977)] this task is to be considered a brightness discrimination.

10. Naïve animals of both sexes collected from the Bay of Naples (Italy), ranging in weight from 200 to $650 \mathrm{~g}$, were held in individual gray plastic tanks $(60 \times 100 \times 50 \mathrm{~cm})$ under described conditions (4). Each experiment started when octopuses were acclimatized to captivity [E. A. Bradley and J. Z. Young, Behav. Biol. 13, 527 (1975)].

11. A demonstrator animal was trained in isolation in experimental tanks. Stimulus balls were inserted simultaneously in the tank at a distance of $80 \mathrm{~cm}$ from the animal and $25 \mathrm{~cm}$ from each other. Both balls, randomly positioned, were simultaneously moved by hand up and down a maximum of 25 times during a trial. The maximum duration for a trial was $1 \mathrm{~min}$, and the interval between trials was $5 \mathrm{~min}$.

12. The demonstrators preferred the red ball to the white. In order to measure any visual preference [N. S. Sutherland and W. R. A. Muntz, Pubbl. Stn Zool. Napoli 31, 109 (1959); J. B. Messenger and G. D. Sanders, Anim. Behav. 20, 580 (1972)] in our experimental conditions, we tested the response of 18 naïve Octopus vulgaris, in isolation, to simultaneous presentation of the red and white ball. A series of five sessions, each consisting of five trials, was conducted on five consecutive days. Trial and intertrial intervals were 1 min and 5 min, respectively; no reward or punishment was given for each trial. After each session a crab was given to each animal as a reward. In the first five trials octopuses chose the ball randomly (first day: 39 red, 34 white in 90 trials). By the fifth day there was a tendency to prefer the red shade (58 red, 16 white in 90 trials).

13. A. Packard, Bull. Inst. Océanogr. Monaco D, 35 (1963).

14. At the end of the observation phase an opaque panel was slid between the two tanks to isolate the observer from the demonstrator. The testing phase of the experiment started 1 hour later.

15. Correct choices were compared to cumulative errors both computed in the five testing trials of the observers [red: 129 (86\%) correct on 150 trials; white: 49 (70\%) correct on 70 trials] and from the fifth to the ninth of the training trials of the demonstrators [red: $77(51 \%)$ correct on 150 trials; white: 36 (51\%) correct on 70 trials].

16. R. W. Mitchell, Perspect. Ethol. 7, 183 (1987).

17. A. N. Meltzoff and M. K. Moore, Science 198, 75 (1977); T. M. Field, R. Woodson, R. Greenberg, D. Cohen, ibid. 218, 179 (1982).

18. P. H. Klopfer, Behaviour 17, 71 (1961); E. R. John P. Chesler, F. Bartlett, I. Victor, Science 159, 1489 (1968); C. K. Tayler and G. S. Saayman, Behaviour 44, 286 (1973); P. D. Weigl and E. V. Hanson, Ecology 61, 213 (1980); R. J. Sanders, J. Comp. Psychol. 99, 197 (1985); B. Palameta and L. Lefebvre, Anim. Behav. 33, 892 (1985); G. B. Biederman, H. A. Robertson, M. Vanayan, J. Exp. 
Anal. Behav 46, 45 (1986): D. Mainardi, M. Mainardi, A. Pasquali, Ethology 72, 191 (1986); M. Anthouard, Behaviour 103, 266 (1987).

19. K Lorenz, Besides The Mirror: A Search For a Natural History of Human Knowledge (Meuthen,
London 1977), p. 113: W. Wyrwicka, Pavlovian J. Biol Sci. 23, 125 (1988)

20. J. Z. Young, Biol. Bull. 180, 200 (1991).

21. We thank G. Salvatore, L. Cariello, and the technical staff of the Stazione Zoologica for their continuous support during this study; and $A$ Packard G. Ferguson, H. Maldonado, J. Messenger, and J. Z. Young for helpful discussions and comments. We are also indebted to the reviewers for their valuable comments and to $F$. Chiarotti.

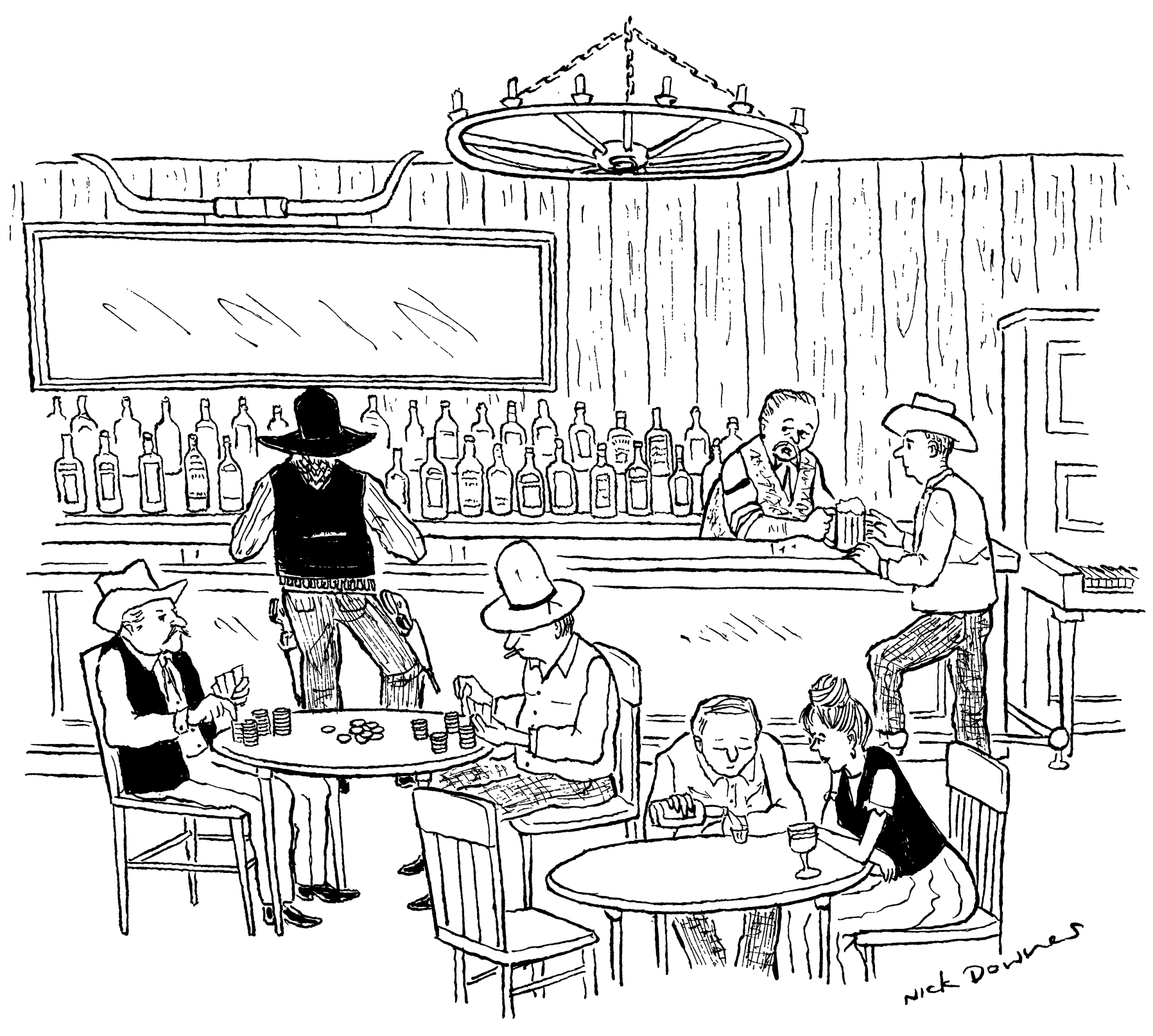

"That's Dead-Eye Dan, known far and wide for his fast gun, mean temper and extra y chromosome." 